\title{
Precalcaneal Congenital Fibrolipomatous Hamartoma
}

\author{
Ji-Hye Yang, M.D., Oun-Jae Park, M.D., Jeong-Eun Kim, M.D., Chong-Hyun Won, M.D., \\ Sung-Eun Chang, M.D., Mi-Woo Lee, M.D., Jee-Ho Choi, M.D., Kee-Chan Moon, M.D. \\ Department of Dermatology, Asan Medical Center, University of Ulsan College of Medicine, Seoul, Korea
}

Precalcaneal congenital fibrolipomatous hamartomas (PCFHs) are characterized clinically by the presence of unilateral or bilateral, asymptomatic nodules in the medial precalcaneal plantar region of the heel. They are skin colored and usually painless nodules. In most patients, the lesions appear within the first few months of life, but they may also be present at birth. Generally PCFHs are benign, but they can grow in proportion to the growth of the infants. Here, we report the case of a 4-month-old boy with a solitary, localized skin-colored nodule on the precalcaneal plantar region of his right heel, diagnosed as a PCFH. (Ann Dermatol 23(1) 92 94, 2011)

\section{-Keywords-}

Precalcaneal congenital fibrolipomatous hamartoma

\section{INTRODUCTION}

Precalcaneal congenital fibrolipomatous hamartomas (PCFHs) are skin-colored, asymptomatic, subcutaneous nodules on the plantar region of the heel. They may be present at birth or develop during infancy. PCFHs are considered to be uncommon and, to our knowledge, none has been reported to date in Korea. We describe here the case of a 4-month-old boy with a PCFH on the medial, plantar aspect of the right heel and discuss its pathogenesis and differential diagnosis.

Received January 12, 2010, Revised April 28, 2010, Accepted for publication May 4, 2010

Corresponding author: Mi-Woo Lee, M.D., Department of Dermatology, Asan Medical Center, University of Ulsan College of Medicine, 388-1 Pungnap-dong, Songpa-gu, Seoul 138-736, Korea. Tel: 82-2-30103460, Fax: 82-2-486-7831, E-mail: miumiu@amc.seoul.kr

\section{CASE REPORT}

A 4-month-old male infant was referred to us with a soft, asymptomatic, skin-colored nodule on the medial aspect of his right heel. This nodule had been present at birth and became larger in size in proportion to the infant's growth. The child was born by cesarean section at gestational age 36 weeks. His birth weight was 1,760 g, but he had no abnormalities at that time except for the physiologic jaundice of newborns. He subsequently showed normal developmental growth. There was no significant family history of PCFHs. Skin examination showed a small, soft, mobile, non-tender, skin-colored nodule, about $1 \mathrm{~cm}$ in diameter, located on the medial, plantar aspect of his right heel, and covered with normal overlying skin (Fig. 1). Ultrasound examination of the plantar region showed a $0.7 \times 0.6 \mathrm{~cm}$ ill-defined, homogeneous, slightly hyperechoic lesion near the subcutaneous layer (Fig. 2). There was no infiltration into the underlying structure. Histopathology of a biopsy specimen taken from the right heel showed multiple, lobulated mature adipose tissues protruding into the dermis and separated by collagenous

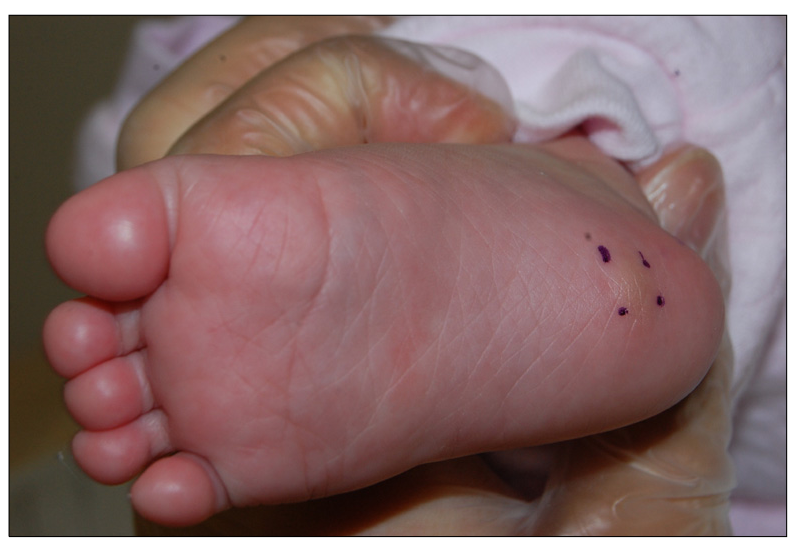

Fig. 1. Photograph of a solitary, $1 \mathrm{~cm}$, soft, skin-colored nodule on the right medial plantar heel surface in a 4-month-old boy. 


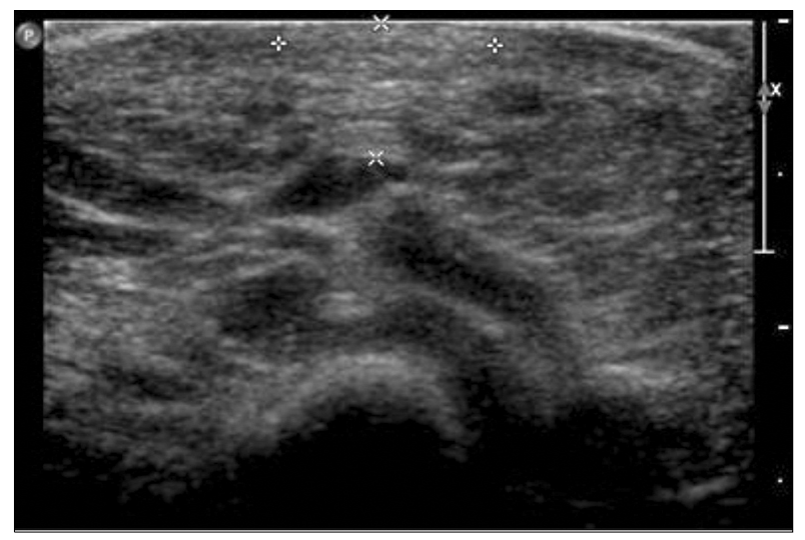

Fig. 2. Ultrasound image showing a $0.7 \times 0.6 \mathrm{~cm}$, ill-defined, homogeneous, slightly hyperechoic lesion near the subcutaneous layer.

fibrous sheaths (Fig. 3). The nodule was diagnosed as a $\mathrm{PCFH}$ and the parents were reassured accordingly.

\section{DISCUSSION}

Since the first description of PCFH in $1990^{1}$, there have been several case reports of them, mostly in the pediatric dermatology literature. Other names for PCFH include bilateral congenital adipose plantar nodules ${ }^{2}$, benign anteromedial plantar nodules of childhood ${ }^{3}$, bilateral congenital fatty heel pads ${ }^{4}$, and hypertrophic infantile pedal papules ${ }^{5}$. Although $\mathrm{PCFH}$ had been considered to be rare, it may also have been underreported.

PCFH usually occurs in otherwise healthy children, but its incidence and inheritance have not been determined. To date, most cases have been sporadic, but PCFH may show a familial pattern ${ }^{6}$. This condition is either present at birth or develops over the first few months after birth. Clinically, these lesions are characterized by asymptomatic nodules with normal overlying skin, and they are typically noticed by parents incidentally. Although PCFHs are usually bilateral and symmetrical, they can also be unilateral, as in our patient. PCFHs may become larger in proportion to the growth of the infant or they may maintain the same size. They usually occur on the anteromedial aspect of the heel, but they can also extend onto the heel area. Histologic examination of these lesions shows mature adipose tissue enveloped in collagenous fibrous sheaths. Ultrasound examination of a PCFH reveals a homogeneous mass that shows similar echogenicity as subcutaneous tissue. The natural history of $\mathrm{PCFH}$ is difficult to determine because most reports have described changes that occur during the first few years of life, without determining long-term outcomes. In most

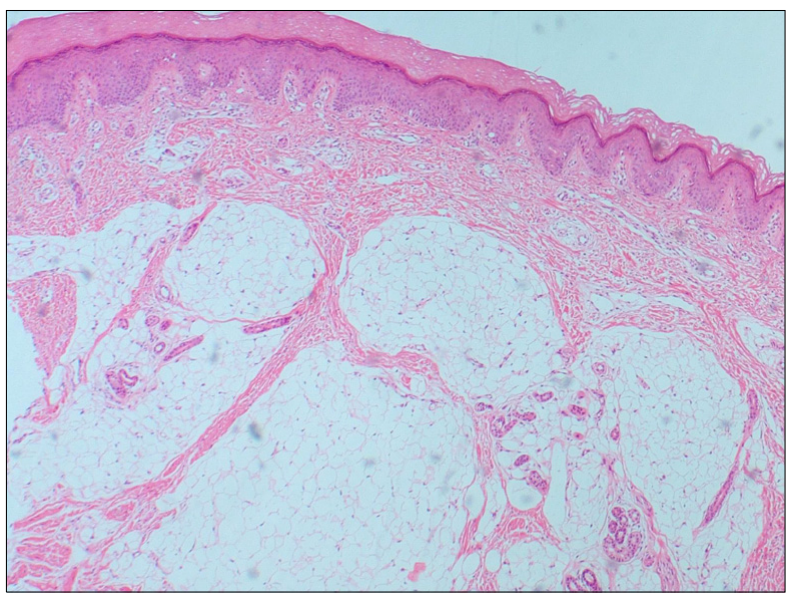

Fig. 3. Light microscope finding showing multiple lobulated mature adipose tissue protruding into the dermis and separated by collagenous fibrous sheaths (H\&E stain, $\times 40)$.

patients, the lesions persisted without significant changes ${ }^{1,6}$, although in some they disappear at $2 \sim 3$ years of age ${ }^{7}$. There have been no reports of malignancy or gait disturbance caused by $\mathrm{PCFH}{ }^{1}$.

The etiology of PCFH is unclear, but there have been several hypotheses. The first hypothesis suggests that $\mathrm{PCFH}$ is associated with a developmental alteration. The fibroconnective trabecular system of the sole develops in the fetal stage and is completely formed during the last months of pregnancy or in the first months after birth ${ }^{1}$. The fetal heel has been observed to exhibit physiologic hypodermic hypertrophy, indicating that a PCFH may result from incomplete regression of fetal tissue ${ }^{8}$. Another hypothesis is that PCFH is caused by fat herniation through defects in the plantar fascia ${ }^{4}$, but these defects have not been observed consistently and the nodules are not reducible. A third hypothesis proposes that PCFH is due to an underlying genetic mechanism caused by autosomal dominant or X-linked inheritance ${ }^{6}$. Larger studies are needed to determine whether there is a genetic mechanism involved and, if so, the mechanism of inheritance.

The differential diagnosis of PCFH includes piezogenic papules, juvenile plantar fibromatosis, nevus lipomatosus superficialis, calcified nodules, congenital solitary histiocytoma, focal dermal hypoplasia, and congenital hemangioma ${ }^{9}$. Piezogenic papules are pressure-induced lesions caused by herniation of fat tissue and are only apparent when patients stand. In contrast, the PCFH nodules are present from birth and experiencing them is not related to gravity. The lesions of juvenile plantar fibromatosis are more indurated than those of $\mathrm{PCFH}$, and histologic examination of the former shows fibrous tissue attached to 
the plantar fascia. The lesion in nevus lipomatosus superficialis are usually distributed linearly on the thigh or hip. Calcified nodules are caused by repeated insertion of needles in the heel to draw blood during the neonatal period. These nodules can be painful, and they show dystrophic cutaneous calcification.

Treatment, other than reassurance, is not required, especially for bilateral nodules. Children with unilateral nodules should be scheduled for re-evaluation after 6 month to determine whether the nodule is getting larger or becoming symptomatic ${ }^{10}$. Ultrasonography may also be performed during the evaluation. The nodule can be excised surgically if tenderness occurs, although this is rare.

In conclusion, $\mathrm{PCFH}$ is a benign condition that does not affect normal activities and usually does not require treatment. Recognition of this condition can avoid unnecessary surgical treatment of the infant and relieve parents' anxieties. Additional case reports and studies are needed to determine the pathologic mechanism and prevalence of PCFH.

\section{REFERENCES}

1. Larralde de Luna M, Ruiz León J, Cabrera HN. Pedal papules in newborn infants. Med Cutan Ibero Lat Am 1990;18:9-12.

2. España A, Pujol RM, Idoate MA, Vázquez-Doval J, Romaní J. Bilateral congenital adipose plantar nodules. Br J Dermatol 2000;142:1262-1264.

3. Jacob Cl, Kumm RC. Benign anteromedial plantar nodules of childhood: a distinct form of plantar fibromatosis. Pediatr Dermatol 2000;17:472-474.

4. Livingstone JA, Burd DA. Bilateral congenital fatty heel pads. Br J Plast Surg 1995;48:252-253.

5. Conde-Taboada A, Mayo E, González B, Pardavila R, De la Torre C, Cruces M. Hypertrophic infantile pedal papules. Pediatr Dermatol 2007;24:339-340.

6. Fangman WL, Prose NS. Precalcaneal congenital fibrolipomatous hamartomas: report of occurrence in half brothers. Pediatr Dermatol 2004;21:655-656.

7. Flann S, Munn SE. Precalcaneal congenital fibrolipomatous hamartoma. Clin Exp Dermatol 2009;34:495-496.

8. Larregue M, Vabres P, Echard P, Cambazard F. Precalcaneal congenital fibrolipomatous hamartoma. Presented at the Fifth International Congress of Pediatric Dermatology, 1996 Sep; Rotterdam, Netherlands.

9. Semadeni BL, Mainetti C, Itin P, Lautenschlager S. Precalcaneal congenital fibrolipomatous hamartomas: report of 3 additional cases and discussion of the differential diagnosis. Dermatology 2009;218:260-264.

10. García-Mata S, Hidalgo-Ovejero A. Anteromedial plantar nodules of the heel in childhood: a variant of the normality? J Pediatr Orthop B 2010;19:108-113. 